\title{
Relação entre a saúde mental de pacientes com câncer avançado em quimioterapia paliativa e seus familiares cuidadores
}

\author{
Relationship between Mental Health of Patients with Advanced \\ Cancer in Palliative Chemotherapy and their Family Caregivers
}

Relación entre la salud mental de pacientes con cáncer avanzado en quimioterapia paliativa y sus cuidadores familiares

Tatiane Ladeira ${ }^{1} \underline{\mathrm{ORCID}}$, Fabiane Grincenkov $2 \underline{\mathrm{ORCID}}$

Universidade Federal de Juiz de Fora

Brasil

Fecha correspondencia:

Recibido: junio 3 de 2019.

Aceptado: enero 14 de 2020.

Forma de citar:

Laderia, T., \& Grincenkov, F. (2020).

Relação entre a Saúde Mental de

pacientes com câncer avançado

em quimioterapia paliativa e seus

familiares cuidadores. Rev. CES Psico,

13(2), 1-17.

\section{Open access}

(c) Copyright

Licencia creative commons

Etica de publicaciones

Revisión por pares

Gestión por Open Journal System

DOl: http://dx.doi.org/10.21615/

cesp.13.2.1

ISSN: 2011-3080

Sobre los autores:

1. Mestre em Psicologia, Psicóloga.

Comparte

\section{Resumo}

Vivenciar uma doença incurável traz à tona o confronto com a finitude, despertando temores e impactando negativamente a saúde mental de pacientes e familiares cuidadores. 0 objetivo deste estudo foi avaliar ansiedade, depressão e qualidade de vida (QV) em pacientes com câncer avançado e os respectivos cuidadores, investigando a associação entre esses fatores. 25 pacientes e 25 cuidadores foram entrevistadas usando WHOQOL Breve, HADS, Escala de sobrecarga ZARIT e questionário sociodemográfico e clínico. Dados foram submetidos à análise descritiva, correlação e comparação. Pacientes e cuidadores apresentaram níveis similares de QV, ansiedade e depressão. No cruzamento entre os grupos, não foram identificadas correlações para a maioria dos domínios de avaliação da QV. Ansiedade ou Depressão. Na análise intragrupos, o domínio psicológico da QV associou-se positivamente a todos os demais domínios avaliados em pacientes e cuidadores. Ansiedade e Depressão associaram-se negativamente à QV em ambos os grupos. Sobrecarga do cuidador relacionou-se inversamente à QV e diretamente à Ansiedade e Depressão dos cuidadores. Ansiedade, Depressão e Sobrecarga são sublinhadas como áreas nas quais pacientes e cuidadores podem se beneficiar de intervenções para melhorar sua QV.

Palavras-chave: Saúde Mental, Pacientes Incuráveis, Familiar Cuidador, Assistência Paliativa, Câncer, Ansiedade, Depressão, Qualidade de Vida.

\section{Abstract}

Facing an incurable disease challenges confrontation with finitude, arousing fears and impacting negatively the mental health of patients and family caregivers. The aim of this study was to evaluate anxiety, depression and quality of life (QoL) in patients with advanced cancer and their caregivers, investigating the association between these factors. 25 patients and 25 caregivers were interviewed using WHOQOL Brief, HADS, ZARIT Burden Interview, and sociodemographic and clinical questionnaire. Data were submitted to 
2. Mestre e Doutora em Saúde. Docente do Programa de PosGraduação em Psicologia da Universidade Federal de Juiz de Fora (UFJF) e da Residencia Multiprofissional em Saúde do Adulto do HU-CAS-UFJF. descriptive analysis, correlation and comparison. Patients and caregivers presented similar levels of QoL, anxiety and depression. At the intersection between groups, no correlations were identified for most domains of QoL, anxiety or depression. In the intragroup analysis, the psychological domain of QoL was associated positively with all other domains evaluated in patients and caregivers. Anxiety and depression were negatively associated with QoL in both groups. Caregiver burden was inversely related to QoL, Anxiety and Depression of caregivers. Anxiety, Depression and Burden are underlined as areas in which patients and caregivers can benefit from interventions to improve their QoL.

Keywords: Mental Health, Incurable Patients, Family Caregiver, Palliative Care, Cancer, Anxiety, Depression, Quality of Life.

\section{Resumen}

Vivir una enfermedad incurable plantea una confrontación con la finitud, despertando temores e impactando negativamente la salud mental de pacientes y familiares cuidadores. El objetivo de este estudio fue evaluar Ansiedad, Depresión y Calidad de Vida (CV) en pacientes con cáncer avanzado y los respectivos cuidadores, investigando la asociación entre dichas variables. Se entrevisto a 25 pacientes y 25 cuidadores mediante el WHOQOL Breve, HADS, Escala de sobrecarga ZARIT y un cuestionario sociodemográfico y clínico. Los datos fueron sometidos a análisis descriptivo, de correlación y comparación. Los pacientes y los cuidadores presentaron niveles similares de CV. Ansiedad y Depresión. En el análisis intergrupos, no se identificaron correlaciones para la mayoría de los dominios de evaluación de la CV, Ansiedad o Depresión. En el análisis intragrupo, el dominio psicológico de la CV se asoció positivamente a todos los demás dominios evaluados en pacientes y cuidadores. La Ansiedad y la Depresión se asociaron negativamente a la CV en ambos grupos. La Sobrecarga del cuidador está inversamente relacionada con la CV y directamente relacionada con la Ansiedad y la Depresión de los cuidadores. La Ansiedad, la Depresión y la Sobrecarga se destacan como áreas en las que los pacientes y los cuidadores pueden beneficiarse de intervenciones para mejorar su CV.

Palabras claves: Salud Mental, Pacientes Incurables, Familiar Cuidador, Cuidados Paliativos, Cáncer, Ansiedad, Depresión, Calidad de Vida.

\section{Introducão}

O mundo contemporâneo vivencia uma tendência global de transição demográfica e epidemiológica ocasionada pelo envelhecimento da população e consequente aumento das doenças crônicas não transmissíveis (DCNT), dentre as quais se destaca o câncer (The Economist, 2015; Worldwide Palliative Care Alliance \& World Health Organization -WHO-, 2014). No Brasil, as DCNT são as mais prevalentes e figuram entre as principais causas de morte da população (Ministério da Saúde, 2014). Esta configuração sanitária revela que um dos maiores problemas a ser enfrentado, tanto pelos profissionais de saúde quanto pelos administradores públicos, é o da oferta de cuidados especializados aos pacientes que se encontram na fase final de suas doenças.

Esses cuidados podem ser compreendidos sob a égide dos Cuidados Paliativos, uma abordagem que objetiva promover a melhor qualidade de vida possível a pacientes e seus familiares durante o enfrentamento às doenças que ameaçam a continuidade da vida, sendo ambos entendidos como uma unidade de cuidados para qual a assistência deve ser direcionada. Para tanto, enfatizam a prevenção e alívio do sofrimento 
Cuidar de um paciente oncológico fora de possibilidade de cura (FPC) constitui uma tarefa complexa e angustiante, uma vez que, com pouco ou nenhum preparo, os familiares cuidadores se veem responsáveis por atividades como cuidados físicos do paciente, administração de medicamentos, entre outros. mediante a identificação precoce, avaliação e tratamento impecáveis da dor e demais problemas de ordem física, psicossocial e espiritual (WHO, 2002).

A vivência de um adoecimento como o câncer pode ser muito cara ao indivíduo, sobretudo quando não há a possibilidade da cura. Nos estágios avançados, encontramos o indivíduo geralmente vulnerável, impotente, com o feroz avanço da doença inscrito em seu corpo e escancarando ao olhar do outro a proximidade do fim. Além disso, mesmo quando não há ciência do diagnóstico ou prognóstico, as perdas cotidianas ou a mera percepção da existência de um adoecimento reverberam em efeitos devastadores na esfera psíquica do paciente (Antonechen \& Dóro, 2016). Como exemplos, podemos destacar o surgimento de sintomas como ansiedade, depressão, anorexia, fadiga e ideações suicidas (Delalibera, Presa, Barbosa, \& Leal, 2015; Götze, Brähler, Gansera, Polze, \& Köhler, 2014).

Embora os sintomas físicos da doença e os efeitos colaterais do tratamento sejam sentidos diretamente pelo paciente, também a família é invitada a confrontar as implicações deste convalescer, partilhando do sofrimento diante da realidade para muitos atemorizante que é o enfrentamento da finitude próxima. Neste panorama, surge a figura do cuidador, designado como tal por instinto, vontade, disponibilidade ou mesmo por um senso de obrigação ou dever para com o familiar adoecido (Genezini, Castro, \& Rossi, 2009).

Cuidar de um paciente oncológico fora de possibilidade de cura (FPC) constitui uma tarefa complexa e angustiante, uma vez que, com pouco ou nenhum preparo, os familiares cuidadores se veem responsáveis por atividades como cuidados físicos do paciente, administração de medicamentos, entre outros. Vivenciam, além disso, tanto o sofrimento diante das perdas do ente querido quanto o relativo às próprias perdas, em função das acentuadas mudanças em sua rotina. Esta situação pode gerar no cuidador uma grande sobrecarga física e emocional, que tende a piorar com a proximidade da morte do paciente (Delalibera et al., 2015; Yoon, Kim, Jung, Kim, \& Kim, 2014).

A literatura tem sido consistente em apontar a sobrecarga do cuidador frequentemente associada à ansiedade, depressão, suporte social limitado e qualidade de vida comprometida (Goldstein, Concato, Fried, Kasl, \& Johnson-Hurzeler, 2004; Song et al., 2012). Além disso, estudos sugerem a sobrecarga vivenciada ao longo da prestação de cuidados como fator de risco para complicações no luto (Ferrario, Cardillo, Vicario, Balzarini, \& Zotti, 2004; Neimeyer \& Burke, 2012).

Prejuízos na saúde mental dos familiares cuidadores, gerados pelos encargos inerentes ao cuidar, podem estar relacionados a um correspondente déficit na saúde mental e na qualidade de vida dos pacientes (Abrahão et al., 2010; Hodges, Humphris, \& Macfarlane, 2005; Rezende et al., 2005). Entretanto, são limitados na literatura estudos que avaliem conjuntamente paciente e cuidador, buscando compreender a relação entre eles, a associação entre as manifestações psicopatológicas de ambos e pontos fortes que os auxiliem no enfrentamento ao câncer avançado - conhecimentos imprescindíveis ao planejamento de uma assistência adequada a este público (Delalibera et al., 2015; Bovero, Leombruni, Miniotti, Rocca, \& Torta, 2016).

Em um cenário ideal de intervenção e suporte aos pacientes com câncer avançado e seus familiares, dever-se-iam seguir os preceitos dos Cuidados Paliativos, fornecendo atenção integral às demandas de ambos. Entretanto, há um grande hiato entre a retórica e as realizações. Na escassez de maiores aparatos para a oferta de 
Considerando tal lacuna na assistência e a demanda por maiores investigações na área para que se possa aprimorar a atenção aos pacientes oncológicos fora de possibilidade de cura e seus familiares cuidadores, busca-se através do presente estudo ampliar o conhecimento acerca da relação entre a saúde mental de ambos mediante a avaliação dos níveis de ansiedade, depressão e qualidade de vida de pacientes e cuidadores, bem como a sobrecarga desses últimos, e a correlação entre esses fatores na amostra pesquisada. cuidados consonantes à filosofia paliativista, prevalece nas redes pública e particular da grande maioria dos centros de referência em oncologia no país um modelo de assistência tradicional, restrita ao ambiente hospitalar, com foco apenas no controle (nem sempre eficaz) dos sintomas físicos dos pacientes. Desprezam-se, dessa forma, tanto aspectos psicossociais do adoecimento quanto a indissolução entre a unidade de cuidados paciente e família, havendo por vezes um total desconhecimento da dinâmica de tais relações e suas implicações nas respostas físicas e psicológicas dos pacientes.

Considerando tal lacuna na assistência e a demanda por maiores investigações na área para que se possa aprimorar a atenção aos pacientes oncológicos fora de possibilidade de cura e seus familiares cuidadores, busca-se através do presente estudo ampliar o conhecimento acerca da relação entre a saúde mental de ambos mediante a avaliação dos níveis de ansiedade, depressão e qualidade de vida de pacientes e cuidadores, bem como a sobrecarga desses últimos, e a correlação entre esses fatores na amostra pesquisada.

\section{Método}

\section{Participantes}

Foram incluídos pacientes oncológicos fora de possibilidade de cura submetidos à quimioterapia com finalidade paliativa e os respectivos familiares cuidadores, considerados nesse estudo como a pessoa apontada pelo paciente como a principal responsável por the prover cuidados, sem estar recebendo reembolso financeiro para este fim. Pacientes e familiares cuidadores foram recrutados simultaneamente, em uma instituição hospitalar de referência na atenção oncológica, localizada no município de Juiz de Fora (Minas Gerais, Brasil), havendo a inclusão de um apenas mediante o aceite do outro em participar da pesquisa. Foi observado, ainda, o critério de idade superior ou igual a 18 anos para a inclusão no estudo.

O processo amostral foi por conveniência, sendo identificados ao longo do período de coleta 33 pacientes aptos a participarem do estudo. Desse total, foram excluídos seis pacientes que não costumavam ir acompanhados de seus cuidadores principais à instituição e dois que recusaram a participação na pesquisa, resultando em um total de 50 participantes ( 25 pacientes e 25 cuidadores) a serem analisados.

\section{Instrumentos}

The World Health Organization Quality of Life (WHOQOL)-Abreviado Instrumento de avaliação da qualidade de vida (QV), cuja versão em português foi desenvolvida por Fleck et al.-(2000). É composto por 26 questões, sendo duas questões gerais que avaliam a percepção da qualidade de vida e satisfação com a saúde e outras 24 representando as 24 facetas que compõem o instrumento original (WHOQOL-100) e presta-se a reunir informações em quatro domínios, a saber: físico, psicológico, relações sociais e meio ambiente. As respostas às 26 questões do instrumento geram pontuações que variam de 1 a 5 conforme o grau de satisfação, sendo os menores escores demonstrativos de pior qualidade de vida, ao passo que os maiores evidenciam uma melhor qualidade de vida (The WHOQOL Group, 1998).

\section{Escala Hospitalar de Ansiedade e Depressão (HADS)}

A escala, que pode ser auto aplicada, possui 14 itens divididos em subescalas de sete itens para depressão (HADS-D) e sete itens para ansiedade (HADS-A). Cada um dos seus itens pode ser pontuado de zero a três, compondo uma pontuação máxima de 21 
pontos para cada escala. Os pontos de corte recomendados para ambas as subescalas são: HADS-ansiedade: sem ansiedade de 0 a 8, com ansiedade $\geq 9$; HADS-depressão: sem depressão de 0 a 8, com depressão $\geq 9$ (Botega, Bio, Zomignani, Garcia Junior, \& Pereira, 1995).

\section{Zarit Burden Interview (Escala de ZARIT)}

O principal objetivo deste instrumento, validado no Brasil por Scazufca (2002), é avaliar os fatores que levam o cuidador à exaustão para, posteriormente, proporcionar atendimentos mais adequados. É composta por 22 itens que medem áreas de atenção como: saúde, vida social e pessoal, situação financeira, emocional, bem-estar e relações interpessoais. Cada item da escala é graduado de 1 a 5, sendo 1 = nunca, 2 = raramente, 3 = algumas vezes; 4 = frequentemente e $5=$ sempre. Quanto maior for a soma final dos pontos, maior será considerado o nível de sobrecarga, que varia de ausente $(\leq 21)$ a severa $(\geq 61)$. Pode ser respondida tanto pelo próprio cuidador quanto em forma de entrevista realizada pelo pesquisador.

\section{Questionário Sociodemográfico e Clínico}

Elaborado pela pesquisadora responsável por este estudo, destinou-se à coleta de dados sociais e demográficos dos cuidadores e pacientes, bem como de dados clínicos desses últimos. A fim de preservar pacientes que porventura não tivessem ciência de seus diagnósticos e prognósticos, tal instrumento foi respondido apenas pelos cuidadores.

\section{Desenho}

A presente pesquisa pode ser descrita como um estudo quantitativo, exploratório, de corte transversal. A coleta de dados foi realizada no período de Fevereiro a Maio de 2017 em uma instituição hospitalar de referência na atenção oncológica localizado no município de Juiz de Fora, MG, Brasil.

\section{Procedimentos}

Pacientes foram triados a partir da verificação dos prontuários de todos aqueles designados a comparecer à sessão de quimioterapia, a fim de identificar a finalidade do tratamento (curativa, adjuvante, prévia, paliativa ou de controle) e a adequação do perfil do paciente aos propósitos da pesquisa. Tal procedimento foi realizado em todas as manhãs da coleta, que aconteceu em média três vezes por semana, nos dias de maior movimento no hospital, em horário que precedia a chegada dos pacientes. A partir da identificação descrita, uma listagem era feita com participantes em potencial para o estudo, os quais eram convidados conforme fossem chegando à instituição.

A aplicação dos instrumentos aos que consentiram a participação no estudo foi realizada pela primeira autora e por uma psicóloga voluntária previamente treinada; ocorreu nas dependências da instituição que sediou a presente pesquisa e se deu de forma individual, sempre que possível em ambiente privado cedido pela equipe de enfermagem e durante o período de sala de espera. Ocasionalmente, alguns dos pacientes foram entrevistados durante a realização do procedimento quimioterápico. Entretanto, entrevistas em tais circunstâncias somente ocorreram quando foi possível assegurar a privacidade do participante, por exemplo, quando não havia outros pacientes realizando o procedimento ao lado do entrevistado. 
Na totalidade dos casos, os pacientes possuíam vínculo em primeiro grau com seus cuidadores, sendo estes cônjuges e/ou companheiros(as) (44\%), filhos ou filhas (32\%), irmãos ou irmãs (16\%), mãe ou pai (8\%) dos pacientes. 0 tempo em que exerciam a função de cuidador variou de um mês a três anos, com maior percentual concentrado no período de um a dois anos (36\%). A dedicação diária do cuidador ou cuidadora ao paciente, por sua vez, variou de uma a 12 horas, sendo que $68 \%$ da amostra relatou prestar cuidados diários ao paciente em tempo integral.

\section{Aspectos Éticos}

Este estudo foi desenvolvido em acordo com as normas da Declaração de Helsinque e foi aprovado pelo Comitê de Ética em Pesquisas com Seres Humanos da Universidade Federal de Juiz de Fora, sob parecer número 1.716.941. Seguindo-se os preceitos da Resolução 466/2012, os pacientes e familiares cuidadores foram informados dos objetivos, riscos e benefícios do estudo e, estando de acordo, assinaram o Termo de Consentimento Livre e Esclarecido - TCLE em sua versão para pacientes ou cuidadores.

\section{Análise dos dados}

Os dados obtidos a partir da aplicação das escalas e do questionário sociodemográfico e clínico foram inicialmente submetidos a análises descritivas, sendo as variáveis quantitativas apresentadas a partir das respectivas médias e desvio padrão, mediana e amplitude e as variáveis qualitativas a partir de sua frequência e porcentagem. Posteriormente, verificou-se a normalidade da distribuição das variáveis selecionadas para os testes correlacionais (Qualidade de Vida, Ansiedade e Depressão em ambos os grupos e a Sobrecarga dos cuidadores) por meio do teste Kolmogorov Smirnov. Como a maioria das distribuições não satisfez os critérios de normalidade e também em função do tamanho reduzido da amostra, optou-se pela realização exclusiva de testes não paramétricos, a fim de uniformizar a análise e garantir maior fidedignidade dos resultados. Dessa forma, as variáveis de interesse acima descritas foram correlacionadas por meio do teste de Spearman e as diferenças na tendência central ao longo do par paciente e cuidador em relação aos níveis de ansiedade, depressão e qualidade de vida por meio do teste Wilcoxon. 0 cálculo dos escores do instrumento WHOQOL Breve foi efetuado a partir do algoritmo disponibilizado pela Organização Mundial de Saúde para este fim. Todas as análises foram realizadas por meio do software SPSS 20.0, presumindo-se um intervalo de confiança superior ou igual a $95 \%$.

\section{Resultados}

\section{Amostra}

Foram entrevistados 25 pacientes e os respectivos 25 cuidadores. As características sociodemográficas da amostra encontram-se sintetizadas na Tabela 1.

Em relação às características clínicas, a localização dos tumores primários nos pacientes entrevistados deu-se, por ordem de frequência: no aparelho digestivo (52\%); mama (16\%); pulmão e pleura (12\%); trato urinário (8\%); ossos e partes moles (4\%); porções ginecológicas (4\%); cabeça e pescoço (4\%). Todos apresentavam metástases, cujo comprometimento variou de um a vários órgãos e tecidos do corpo. 0 tratamento predominante, além da quimioterapia - a que todos haviam se submetido - foi a cirurgia (76\%). $28 \%$ foram submetidos também à radioterapia e outros $8 \%$ a tratamentos alternativos. $64 \%$ dos pacientes foram diagnosticados pelo Sistema Único de Saúde (SUS) brasileiro e o restante (36\%) pela rede privada de saúde.

$\mathrm{Na}$ totalidade dos casos, os pacientes possuíam vínculo em primeiro grau com seus cuidadores, sendo estes cônjuges e/ou companheiros(as) (44\%), filhos ou filhas (32\%), irmãos ou irmãs (16\%), mãe ou pai (8\%) dos pacientes. 0 tempo em que exerciam a função de cuidador variou de um mês a três anos, com maior percentual concentrado no período de um a dois anos (36\%). A dedicação diária do cuidador ou cuidadora ao paciente, por sua vez, variou de uma a 12 horas, sendo que $68 \%$ da amostra relatou prestar cuidados diários ao paciente em tempo integral. 19 cuidadores (76\%) relataram morar com o paciente à época da realização da coleta. Todos os cuidadores residiam na mesma cidade que o paciente. Desse total, dois (8\%) relataram haver se 
mudado de cidade em função do adoecimento do familiar. A maioria dos cuidadores não trabalhava no momento (64\%). Desses, cinco (20\%) encontravam-se afastados do trabalho em função do adoecimento de seu familiar. $76 \%$ dos pacientes não trabalhavam no momento da pesquisa. Dos que declararam trabalhar, apenas um (4\%) referiu possuir vínculo empregatício formal.

Tabela 1. Perfil sociodemográfico dos cuidadores e pacientes entrevistados

\begin{tabular}{|c|c|c|c|c|}
\hline Variável & \multicolumn{2}{|c|}{ Cuidador } & \multicolumn{2}{|c|}{ Paciente } \\
\hline \multicolumn{5}{|l|}{ Idade } \\
\hline Média (DP) & \multicolumn{2}{|c|}{$49,16(13,87)$} & \multicolumn{2}{|c|}{$56,08(14,16)$} \\
\hline \multirow[t]{2}{*}{ Mediana (Amplitude) } & \multicolumn{2}{|c|}{50 (22 a 72) } & \multicolumn{2}{|c|}{55 (25 a 87) } \\
\hline & $N$ & $\%$ & $N$ & $\%$ \\
\hline \multicolumn{5}{|l|}{ Sexo } \\
\hline Masculino & 6 & 24,0 & 12 & 48,0 \\
\hline Feminino & 19 & 76,0 & 13 & 52,0 \\
\hline \multicolumn{5}{|l|}{ Cidade } \\
\hline Juiz de Fora & 14 & 56,0 & 14 & 56,0 \\
\hline Outras & 11 & 44,0 & 11 & 44,0 \\
\hline \multicolumn{5}{|l|}{ Situação conjugal } \\
\hline Casado/amigado & 17 & 68,0 & 20 & 80,0 \\
\hline Solteiro & 6 & 24,0 & 1 & 4,0 \\
\hline Viúvo & 2 & 8,0 & 3 & 12,0 \\
\hline Divorciado/Separado & 0 & 0,0 & 1 & 4,0 \\
\hline \multicolumn{5}{|l|}{ Cor } \\
\hline Branco & 16 & 64,0 & 17 & 68,0 \\
\hline Pardo & 5 & 20,0 & 5 & 20,0 \\
\hline Negro & 4 & 16,0 & 3 & 12,0 \\
\hline \multicolumn{5}{|l|}{ Escolaridade } \\
\hline Não alfabetizado & 0 & 0,0 & 1 & 4,0 \\
\hline Nível Fundamental Incompleto & 8 & 32,0 & 13 & 52,0 \\
\hline Nível Fundamental Completo & 2 & 8,0 & 1 & 4,0 \\
\hline Ensino Médio Completo & 10 & 40,0 & 6 & 24,0 \\
\hline Superior Completo & 3 & 12,0 & 3 & 12,0 \\
\hline Pós-Graduação & 2 & 8,0 & 1 & 4,0 \\
\hline \multicolumn{5}{|l|}{ Situação ocupacional } \\
\hline Trabalhando & 9 & 36,0 & 6 & 24,0 \\
\hline Do lar & 6 & 24,0 & 2 & 8,0 \\
\hline Afastado do trabalho & 5 & 20,0 & 8 & 32,0 \\
\hline Aposentado & 3 & 12,0 & 8 & 32,0 \\
\hline Desempregado & 2 & 8,0 & 1 & 4,0 \\
\hline \multicolumn{5}{|l|}{ Religião } \\
\hline Católica & 13 & 52,0 & 18 & 72,0 \\
\hline Evangélica & 5 & 20,0 & 2 & 8,0 \\
\hline Espírita & 3 & 12,0 & 3 & 12,0 \\
\hline Outras religiões & 3 & 12,0 & 1 & 4,0 \\
\hline Sem religião & 1 & 4,0 & 1 & 4,0 \\
\hline
\end{tabular}


Avaliação da Qualidade de Vida, Ansiedade e Depressão de pacientes e cuidadores No que concerne à Qualidade de Vida (QV), pudemos constatar que, dentre os quatro domínios da avaliação, cuidadores alcançaram a maior média no Domínio Físico $(69,88$ pontos; D.P. $=16,46)$ e a menor no domínio Relações Sociais $(58,70$ pontos; D.P. $=23,50)$. Pacientes, por sua vez, alcançaram a maior média no domínio Meio Ambiente $(63,28$ pontos; D.P. $=11,77)$ e a menor no domínio Físico da avaliação $(52,83$ pontos; D.P. $=17,87)$. Nas duas questões gerais de avaliação da QV, cuidadores e pacientes apresentaram médias semelhantes para a percepção da qualidade de vida $(3,72 ;$ D.P. $=0,98$ e 3,72; D.P. $=1,02$, respectivamente $)$ e pacientes alcançaram média inferior aos cuidadores na questão sobre satisfação com a saúde $(3,08$; D.P.= 1,18 e 3,72; D.P. 0,79 , respectivamente). A média relativa aos demais domínios, bem como a mediana e a amplitude dos dados encontram-se discriminadas na Tabela 2. enquanto as médias alcançadas para cada faceta do WHOQOL são apresentadas na Figura 1.

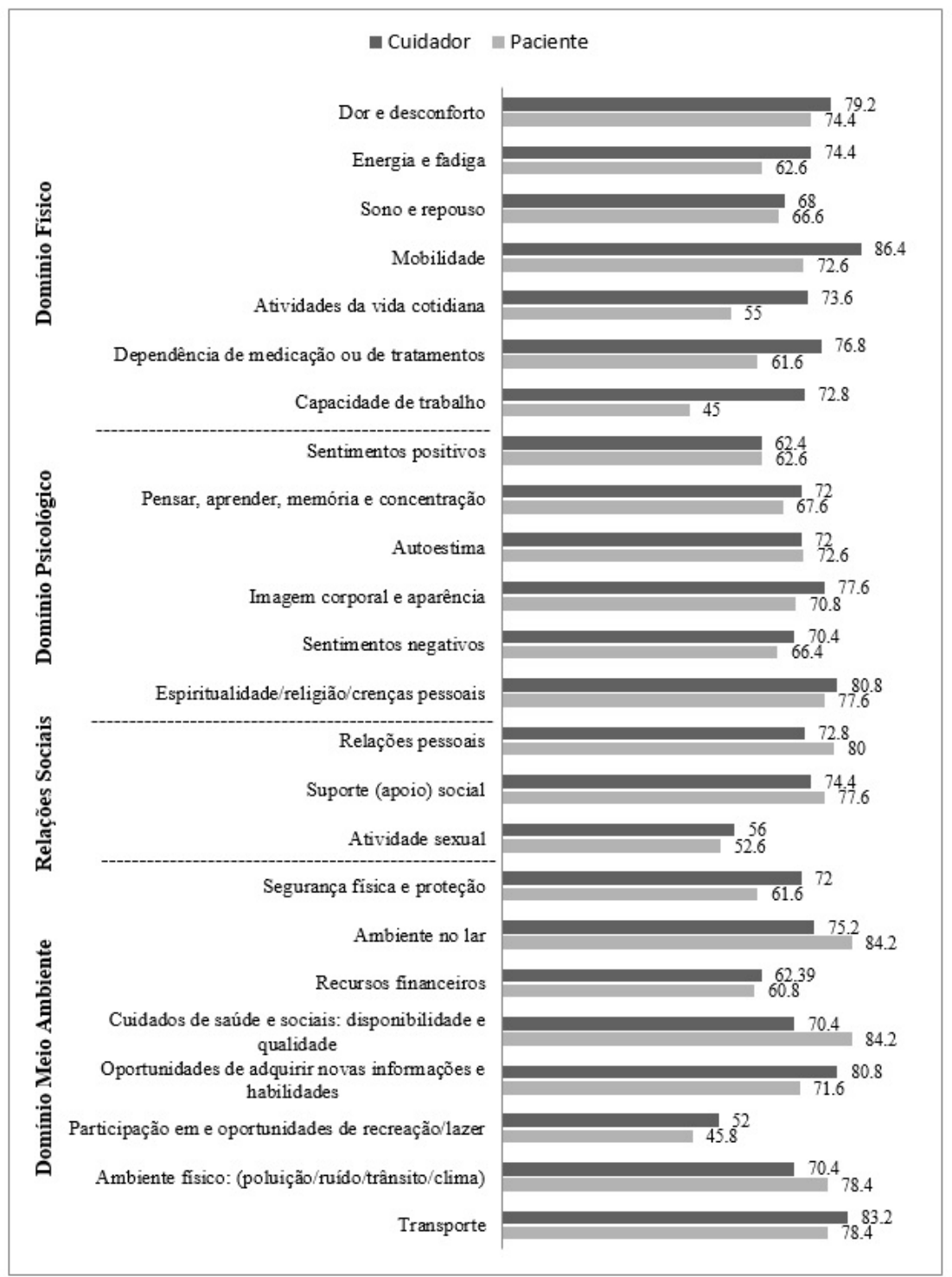

Figura 1. Médias alcançadas por pacientes e cuidadores em cada faceta do WHOQOL 
A correção da escala HADS conforme os critérios preestabelecidos pelos autores (Botega et. al., 1995) possibilitou classificar $48 \%$ dos cuidadores e $52 \%$ dos pacientes com sinais indicativos de Ansiedade e $28 \%$ dos cuidadores e $36 \%$ dos pacientes com sinais indicativos de Depressão. Resultados referentes à análise descritiva dos escores obtidos a partir da aplicação da escala podem ser observados na Tabela 2.

A análise da diferença na tendência central dos pares ao longo das variáveis Ansiedade, Depressão e QV, realizada por meio do teste Wilcoxon, apontou para uma grande semelhança entre as distribuições, uma vez que foi observada diferença estatisticamente significativa apenas para o domínio físico da avaliação da qualidade de vida, com pacientes apresentando menores escores no domínio físico em comparação aos cuidadores $(Z=-3,13 ; p=0,002)$ (Tabela 2).

Tabela 2. Análise descritiva da Qualidade de Vida (WHOQOL Breve), Ansiedade e Depressão (HADS) e comparação entre escores grupais para pacientes e cuidadores

\begin{tabular}{|c|c|c|c|c|c|c|}
\hline \multirow{2}{*}{ Domínios } & \multicolumn{2}{|c|}{ Média (DP) } & \multicolumn{2}{|c|}{ Mediana (Amplitude) } & \multicolumn{2}{|c|}{ Wilcoxon } \\
\hline & Cuidador & Paciente & Cuidador & Paciente & $Z$ & $p$ \\
\hline Percepção da QV & $3,72(0,98)$ & $3,72(1,02)$ & 4,0 (1 a 5) & 4,0 (1 a 5) & $-0,10$ & 0,918 \\
\hline $\begin{array}{l}\text { Satisfação com a } \\
\text { saúde }\end{array}$ & $3,72(0,79)$ & $3,08(1,18)$ & $4,0(2$ a 5) & 3,5 (1 a 5) & $-1,85$ & 0,064 \\
\hline Domínio Físico & $69,88(16,46)$ & $52,83(17,87)$ & 75,0 (36 a 93) & 50,0 (11 a 89) & $-3,13$ & 0,002 \\
\hline Domínio Psicológico & $65,40(21,37)$ & $61,81(17,49)$ & $66,9(17$ a 96$)$ & $66,6(21$ a 88$)$ & $-0,56$ & 0,572 \\
\hline Relações Sociais & $58,70(23,50)$ & $62,50(20,26)$ & $66,6(8$ a 100$)$ & $58,3(25$ a 100$)$ & $-0,18$ & 0,856 \\
\hline Meio Ambiente & $63,45(12,00)$ & $63,28(11,77)$ & $65,6(38$ a 81$)$ & $64,0(41$ a 81$)$ & $-0,35$ & 0,725 \\
\hline Ansiedade & $8,57(5,33)$ & $9,29(4,55)$ & $8,0(0$ a 20$)$ & 9,0 (3 a 19) & $-0,43$ & 0,661 \\
\hline Depressão & $6,30(4,36)$ & $6,54(4,52)$ & $5,0(0$ a 17$)$ & $5,0(1$ a 17$)$ & $-0,29$ & 0,770 \\
\hline
\end{tabular}

Com relação à Sobrecarga do cuidador, mensurada pela escala ZARIT, 14 (56\%) cuidadores receberam a classificação sem sobrecarga e 11 (44\%) reportaram sinais significativos de sobrecarga, que variou de intensa (seis cuidadores) a moderada (cinco cuidadores). A pontuação alcançada pelos cuidadores ficou entre 24 e 90 pontos, com mediana 45 e média de 48,52 pontos ( $D P=17,90)$.

\section{Relação entre a Qualidade de Vida dos pacientes e familiares cuidadores}

O cruzamento entre os escores relativos aos domínios do WHOQOL para pacientes e cuidadores evidenciou a existência de correlações estatisticamente significativas tanto na análise intergrupos quanto nas análises intragrupos.

No primeiro caso, observou-se a ocorrência de relação positiva e significativa entre as seguintes variáveis: satisfação com a saúde em ambos os grupos $(\rho=0,465 ; p=0,019)$; satisfação com a saúde em pacientes e domínio físico do WHOQOL em cuidadores ( $\rho=$ 0,433; $p=0,030$ ); domínio relações sociais em pacientes e domínio meio ambiente em cuidadores $(\rho=0,523 ; p=0,011)$; e, finalmente, domínio meio ambiente em ambos os grupos $(\rho=0,500 ; p=0,11)$.

As análises intragrupos, por sua vez, revelaram diversas associações com significância estatística, todas positivas, conforme pode ser observado na Tabela 3 . Na análise restrita ao grupo de pacientes, as melhores correlações observadas foram 
entre a Percepção da qualidade de vida e os domínios relações sociais $(\rho=0,617, p=$ $0,001)$ e psicológico $(\rho=0,667 ; p=0,001)$ do WHOQOL. No grupo de cuidadores, as correlações de maior grau foram observadas entre domínio psicológico e as variáveis satisfação com a saúde $(\rho=0,626 ; p=0,001)$, domínio físico $(\rho=0,660 ; p<0,001)$ e domínio meio ambiente $(\rho=0,611 ; p=0,001)$ da avaliação da QV.

\section{Relação entre a Sobrecarga do familiar cuidador e a Qualidade de Vida dos pacientes e familiares cuidadores}

Não foram observadas correlações estatisticamente significativas entre os índices de Sobrecarga do cuidador e os escores de QV dos pacientes. Não obstante, tais índices relacionaram-se negativa e significativamente à percepção da qualidade de vida $(\rho=-0,524 ; p=0,007)$ e aos domínios psicológico $(\rho=-0,483 ; p=0,015)$ e relações sociais ( $\rho=-0,484 ; p=0,014)$ da avaliação da QV nos cuidadores, indicando que, quanto mais elevados os índices de sobrecarga, maior comprometimento pode ser observado nesses domínios (Tabela 3).

Tabela 3. Matriz da correlação entre os resultados do WHOQOL para pacientes e cuidadores e entre os escores da escala ZARIT e os resultados do WHOQOL em ambos os grupos

\begin{tabular}{|c|c|c|c|c|c|c|c|c|c|c|c|c|c|}
\hline & & \multicolumn{6}{|c|}{ Pacientes } & \multicolumn{6}{|c|}{ Cuidadores } \\
\hline & & $Q V$ & SS & WQ1 & WQ2 & WQ3 & WQ4 & $Q V$ & SS & WQ1 & WQ2 & WQ3 & WQ4 \\
\hline \multirow{6}{*}{ 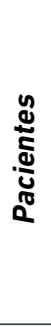 } & $Q V$ & 1,000 & & & & & & & & & & & \\
\hline & SS & $0,583^{* *}$ & 1,000 & & & & & & & & & & \\
\hline & WQ1 & 0,215 & 0,087 & 1,000 & & & & & & & & & \\
\hline & WQ2 & $0,667^{* *}$ & $0,498^{\circ}$ & 0,250 & 1,000 & & & & & & & & \\
\hline & WQ3 & $0,617^{* *}$ & $0,517^{* *}$ & $-0,045$ & $0,554^{* *}$ & 1,000 & & & & & & & \\
\hline & WQ4 & $0,536^{* *}$ & 0,077 & 0,160 & $0,476^{\circ}$ & $0,410^{*}$ & 1,000 & & & & & & \\
\hline \multirow{7}{*}{ 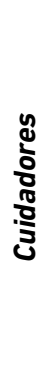 } & $Q V$ & 0,093 & 0,202 & $-0,010$ & $-0,044$ & 0,107 & 0,306 & 1,000 & & & & & \\
\hline & SS & 0,040 & $0,465^{*}$ & 0,005 & 0,161 & 0,094 & 0,068 & $0,509^{* *}$ & 1,000 & & & & \\
\hline & WQ1 & 0,306 & $0,433^{\circ}$ & 0,040 & 0,099 & 0,368 & 0,299 & $0,544^{*}$ & $0,545^{* *}$ & 1,000 & & & \\
\hline & WQ2 & 0,022 & 0,326 & $-0,173$ & 0,031 & 0,227 & 0,281 & $0,553^{* *}$ & $0,626^{* *}$ & $0,660^{* *}$ & 1,000 & & \\
\hline & WQ3 & 0,258 & 0,261 & $-0,227$ & 0,264 & 0,367 & 0,199 & 0,296 & 0,244 & 0,345 & 0,506 & 1,000 & \\
\hline & WQ4 & 0,300 & 0,250 & 0,011 & 0,289 & $0,523^{* *}$ & $0,500^{\circ}$ & $0,595^{* *}$ & 0,331 & $0,549^{* *}$ & $0,611^{* *}$ & $0,562^{* *}$ & 1,000 \\
\hline & ZARIT & $-0,006$ & $-0,135$ & $-0,060$ & $-0,005$ & $-0,244$ & $-0,246$ & $-0,524$ & $-0,123$ & $-0,316$ & $-0,483^{*}$ & $-0,484$ & $-0,374$ \\
\hline & $\begin{array}{l}\text { rrelação é s } \\
\text { orrelação é } \\
\text { Percepção } \\
\text { Satisfação }\end{array}$ & $\begin{array}{l}\text { significante at } \\
\text { significante a } \\
\text { da Qualidade } \\
\text { com a Saúde }\end{array}$ & $\begin{array}{l}\text { té } 0.05 \\
\text { até } 0.01 \\
\text { e de Vida } \\
\text { e }\end{array}$ & $\begin{array}{l}\text { WQ1 - Dor } \\
\text { WQ2 - Dor } \\
\text { WQ3 - Dor } \\
\text { WQ4 - Dor }\end{array}$ & $\begin{array}{l}\text { mínio Físico } \\
\text { omínio Psicol } \\
\text { omínio Relaç } \\
\text { mínio Meio }\end{array}$ & $\begin{array}{l}\text { lógico do Wh } \\
\text { cōes Sociais } \\
\text { Ambiente do }\end{array}$ & $\begin{array}{l}\text { HOQOL } \\
\text { do WHOQOL } \\
\text { O WHOQOL }\end{array}$ & & & & & & \\
\hline
\end{tabular}

\section{Correlação entre Ansiedade, Depressão e Qualidade de Vida dos pacientes e seus familiares cuidadores}

$\mathrm{Na}$ análise intragrupo referente aos pacientes, a ansiedade relacionou-se negativa e significativamente à percepção da qualidade de vida $(\rho=-0,628 ; p=0,001)$ e aos domínios físico $(\rho=-0,457 ; p=0,022)$ e psicológico $(\rho=-0,647 ; p<0,001)$ do WHOQOL. Ainda nesse grupo, observou-se correlação significativa e negativa entre Depressão e os domínios físico ( $\rho=-0,414 ; p=0,040)$, psicológico $(\rho=-0,457 ; p=0,022)$ e meio ambiente $(\rho=-0,471 ; p=0,018)$ da avaliação da $Q$ V. 
Correlacionando os escores de pacientes e cuidadores, as únicas associações com significância estatística observadas foram entre a avaliação do domínio meio ambiente pelos pacientes e os sinais de Ansiedade $(\rho=-0,460 ; p=0,021)$ e Depressão $(\rho=-0,426$; $p=0,034)$ rastreados nos cuidadores, ambas com direcionamento negativo.

A análise restrita ao grupo de cuidadores, por sua vez, evidenciou correlações negativas e significativas entre a Ansiedade do cuidador e os domínios físico ( $\rho=-0,475$; $p=0,016)$, psicológico $(\rho=-0,665 ; p<0,001)$ e meio ambiente $(\rho=-0,595 ; p=0,002)$ do WHOQOL, bem como a percepção da qualidade de vida $(\rho=-0,679 ; p<0,001)$. A variável Depressão relacionou-se a todos os domínios da avaliação da QV dos cuidadores, com destaque para os domínios psicológico $(\rho=-0,770 ; p<0,001)$ e físico $(\rho=-0,745$; $p<0,001)$, onde foram constatadas as correlações de maior grau. Não houve relação significativa entre a Ansiedade e a Depressão de pacientes e a qualidade de vida dos familiares cuidadores (Tabela 4).

Tabela 4. Matriz da correlação entre escores da HADS e domínios do WHOQOL para pacientes e cuidadores

\begin{tabular}{|c|c|c|c|c|c|c|c|c|c|c|c|c|c|}
\hline & & \multicolumn{6}{|c|}{ Pacientes } & \multicolumn{6}{|c|}{ Cuidadores } \\
\hline & & $Q V$ & SS & WQ1 & WQ2 & WQ3 & WQ4 & $Q V$ & SS & WQ1 & WQ2 & WQ3 & WQ4 \\
\hline \multirow{2}{*}{ 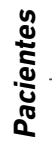 } & HADS-A & $-0,628^{* *}$ & $-0,344$ & $-0,457^{*}$ & $-0,647^{* *}$ & $-0,322$ & $-0,314$ & 0,247 & $-0,059$ & $-0,082$ & 0,103 & 0,168 & 0,068 \\
\hline & HADS-D & $-0,324$ & $-0,106$ & $-0,414^{*}$ & $-0,457^{*}$ & $-0,278$ & $-0,471^{*}$ & 0,021 & $-0,068$ & $-0,289$ & 0,020 & 0,181 & $-0,057$ \\
\hline \multirow{3}{*}{$\begin{array}{l}\text { ! } \\
\vdots \\
0 \\
\frac{\pi}{0} \\
0 \\
0\end{array}$} & HADS-A & $-0,031$ & $-0,217$ & $-0,083$ & 0,012 & $-0,155$ & $-0,460^{*}$ & $-0,679^{* *}$ & $-0,393$ & $-0,475^{*}$ & $-0,665^{* *}$ & $-0,243$ & $-0,595^{* *}$ \\
\hline & HADS-D & $-0,064$ & $-0,234$ & $-0,083$ & $-0,094$ & $-0,326$ & $-0,426^{*}$ & $-0,693^{* *}$ & $-0,432$ & $-0,745^{*}$ & $-0,770^{* *}$ & $-0,477^{*}$ & $-0,674^{*}$ \\
\hline & ZARIT & $-0,006$ & $-0,135$ & $-0,060$ & $-0,005$ & $-0,244$ & $-0,246$ & $-0,524^{* *}$ & $-0,123$ & $-0,316$ & $-0,483$ & $-0,484^{*}$ & $-0,374$ \\
\hline & $\begin{array}{l}\text { Percepção da Q } \\
\text { Satisfação com a }\end{array}$ & $\begin{array}{l}\text { ificante até } 0 \\
\text { uualidade de } \\
\text { a Saúde }\end{array}$ & & $\begin{array}{l}\text { Q3 - Domíni } \\
\text { Q4 - Domíni }\end{array}$ & $\begin{array}{l}\text { io Relações So } \\
\text { io Meio Ambie }\end{array}$ & $\begin{array}{l}\text { ciais do WHC } \\
\text { ente } \mathrm{WHO}\end{array}$ & $\begin{array}{l}\mathrm{L} \\
\mathrm{DQOL} \\
\mathrm{QOL}\end{array}$ & & & & & & \\
\hline
\end{tabular}

\section{Correlação entre Ansiedade, Depressão e Sobrecarga dos pacientes e familiares cuidadores}

Não foram identificadas correlações significativas entre os sinais indicativos de Ansiedade e Depressão quando comparados os escores de pacientes e cuidadores ( $\rho=-0,099 ; p=0,638$ e $\rho=0,246 ; p=0,237$, respectivamente). A Sobrecarga do cuidador relacionou-se positivamente aos sinais de Ansiedade $(\rho=0,688 ; p<0,001)$ e Depressão $(\rho=0,608 ; p=0,001)$ dos próprios cuidadores e não se associsou à Ansiedade e Depressão rastreada nos pacientes. $(\rho=-0,187 ; p=0,371$ e $\rho=0,044$; $p=0,836$, respectivamente).

\section{Discussão}

A realização do presente estudo nos permitiu explorar, para a amostra estudada, aspectos relativos à Saúde Mental e Qualidade de Vida de pacientes com câncer avançado e seus familiares cuidadores, bem como a correlação entre tais dados, respondendo às nossas principais questões de pesquisa.

No que diz respeito à $Q V$, pacientes e cuidadores apresentaram níveis similares para a maioria dos domínios, com distinções significativas restritas à satisfação com a 
Destacamos o comprometimento apresentado pelos cuidadores no domínio relações sociais, onde, mesmo sem significância estatística, identificou-se menor satisfação que a apresentada pelos pacientes, especialmente na faceta suporte social. Esse resultado corrobora o fato de que, muitas vezes, cuidadores se esforçam em prover o paciente com um cuidado adequado sem, ao mesmo tempo, ter uma rede de suporte para the auxiliar, implicando em grande ônus para sua saúde mental e tornando-os altamente suscetíveis aos efeitos da sobrecarga relacionada ao cuidar (Delalibera et al., 2015; Wasner, Paal, \& Borasio, 2013). saúde e ao domínio físico, onde pacientes apresentaram menores índices em relação aos cuidadores. A este respeito, cabe destacar que os pacientes entrevistados pontuaram em um nível bom, próximo ao ótimo, na faceta sobre dor e desconforto, sugerindo um manejo eficaz dos sintomas comuns à fase avançada do adoecimento para a amostra pesquisada. Não obstante, a limitação em atividades cotidianas e capacidade para o trabalho foram os principais responsáveis pela baixa média encontrada para o domínio físico. Resultado semelhante é reportado por Yamashita-(2014), que aponta o campo atividades de vida diária como o de maior impacto na QV de pacientes FPC. Zhang, Nilsson e Prigerson-(2012) destacam um adequado manejo de sintomas, interação satisfatória com a equipe de saúde e prestação de cuidados no âmbito religioso ou espiritual como associados a maiores níveis de QV em pacientes incuráveis. Na via inversa, tratamentos invasivos realizados recentemente impactaram negativamente nessa variável.

Destacamos o comprometimento apresentado pelos cuidadores no domínio relações sociais, onde, mesmo sem significância estatística, identificou-se menor satisfação que a apresentada pelos pacientes, especialmente na faceta suporte social. Esse resultado corrobora o fato de que, muitas vezes, cuidadores se esforçam em prover o paciente com um cuidado adequado sem, ao mesmo tempo, ter uma rede de suporte para the auxiliar, implicando em grande ônus para sua saúde mental e tornando-os altamente suscetíveis aos efeitos da sobrecarga relacionada ao cuidar (Delalibera et al., 2015; Wasner, Paal, \& Borasio, 2013).

Em nosso estudo, parcela significativa dos cuidadores (44\%) apresentou sobrecarga moderada a intensa. Fato que pode ser justificado pela elevada carga horária de dedicação diária aos pacientes, na maior parte dos casos superior a 12 horas por dia, e também ao tempo em que vinham exercendo tal cuidado, uma vez que a maioria encontrava-se há pelo menos um ano acompanhando o paciente em exaustivas rotinas de internações, exames, consultas, quimioterapia e outros procedimentos.

Yoon et al. (2014) investigaram fatores associados à sobrecarga em 64 cuidadores de pacientes com câncer terminal sul coreanos, no intuito de identificar aqueles que seriam passíveis de modificação. A partir de sua análise, reportam maior dedicação diária ao cuidado, menor número de visitas de outros familiares, funcionamento familiar precário e baixa autoestima como fatores modificáveis associados à sobrecarga do cuidador, ao passo que baixo rendimento mensal e relação conjugal com o paciente são apontados como fatores não modificáveis associados ao aumento nesta variável. A sobrecarga do cuidador, embora amplamente estudada e bem descrita na literatura, é ainda pouco valorizada no sentido de poucas unidades de atenção em oncologia desenvolverem e incorporarem estratégias de manejo à sobrecarga do cuidador ao cotidiano do serviço.

Contrariando a hipótese inicial do estudo - e os achados de Li, Xu, Zhou e Loke (2016) - poucas correlações foram verificadas entre os domínios de avaliação da QV no cruzamento entre os grupos. Discordância semelhante foi reportada por Lee et al.-(2016), que avaliando 178 pares de pacientes com câncer avançado e os respectivos cuidadores não encontraram associação entre a QV de ambos ( $p=0,227)$. Entretanto, enquanto nesse último estudo a ausência de correlação é creditada pelos autores à utilização de instrumentos distintos, em nosso estudo utilizamos o mesmo instrumento para acessar a QV de ambos, sendo então mais provável que a falta de associação deva-se ao tamanho limitado da amostra. Sobre o estudo de Li et al.-(2016), destacamos que o fato de os cuidadores participantes do estudo serem todos cônjuges 
Pág 13

Destacamos achados de relevância clínica e convergentes com o esperado, tais como a associação da sobrecarga à QV de cuidadores; dos níveis de ansiedade e depressão à sobrecarga; e, particularmente, a associação forte e altamente significativa entre ansiedade e depressão e a QV de pacientes e cuidadores. dos pacientes pode indicar um maior envolvimento no cuidado e haver favorecido a maior congruência entre o par paciente e cuidador reportada pelos autores.

Em nossa pesquisa, aproximadamente a metade dos pacientes e cuidadores apresentou sinais de ansiedade e cerca de um terço da amostra reportou sinais de depressão, sem diferenças significativas entre os grupos. No entanto, o cruzamento dos dados relativos à ansiedade e depressão entre os grupos de cuidadores e pacientes não apontou a existência de correlações positivas, indicando não haver, para a presente amostra, associação entre tais variáveis.

Nosso resultado contrasta com o reportado por Hodges et al. (2015), que através de uma meta-análise conduzida a partir de 21 amostras independentes de pacientes oncológicos e seus respectivos cuidadores - com n variando de 15 a 288 díades corroboraram a existência de uma associação positiva entre o distress psicológico da díade paciente-cuidador, que parece aumentar em função da progressão da doença. No entanto, converge com os achados dos mesmos autores no que diz respeito à comparação entre os grupos, uma vez que não houve distinção significativa entre os níveis de ansiedade e depressão experimentados por pacientes e cuidadores em ambos os estudos.

Prosseguindo na análise, destacamos achados de relevância clínica e convergentes com o esperado, tais como a associação da sobrecarga à QV de cuidadores; dos níveis de ansiedade e depressão à sobrecarga; e, particularmente, a associação forte e altamente significativa entre ansiedade e depressão e a QV de pacientes e cuidadores. Nesse sentido, pontuamos (a) sobrecarga, ansiedade e depressão como fatores negativamente relacionados à QV de cuidadores - sendo que o distress psicológico destes pode afetar negativamente a satisfação de pacientes com relação ao ambiente em que vivem; e (b) a ansiedade e depressão dos pacientes como fatores preponderantemente relacionados à QV destes, com maiores níveis de distress psicológico associados a menores níveis de satisfação em relação à QV. Este resultado dialoga com os achados de Li et al.-(2016), que identificaram ansiedade e depressão como o fator independente de maior influência na qualidade de vida de 131 pacientes com câncer avançado e seus cônjuges cuidadores.

Ao contrário do que se esperava, no entanto, a sobrecarga não se relacionou de forma estatisticamente significativa a nenhum dos domínios da QV dos pacientes. Tal resultado contrasta com os achados de Wasner et al. (2013) e Borges et al.-(2017), uma vez que, em ambos os estudos, identificou-se associação negativa entre os níveis de qualidade de vida dos pacientes e a sobrecarga dos respectivos cuidadores.

Considerando a consistência nos achados desses estudos mesmo diante de uma grande variação amostral, torna-se mais provável que, em nossa pesquisa, a ausência de associação entre a sobrecarga do cuidador e a qualidade de vida do paciente deva-se ao fato de esses últimos encontrarem-se com a QV pouco comprometida. Ressaltamos, para tanto, que pacientes com maior comprometimento da QV, comum em pacientes menos assistidos e/ou nas etapas finais do adoecimento, costumam demandar mais de seus cuidadores, levando-os a apresentar maiores níveis de sobrecarga relacionada ao cuidado (Delalibera et al., 2015).

Nossos achados chamam a atenção para a importância da atuação da Psicologia junto aos pacientes com câncer avançado e os respectivos familiares cuidadores, uma vez que, para a amostra em questão, as condições psicológicas mostraram estar 
Pág 14

Convém pontuar que o grau de magnitude moderado a forte encontrado em várias correlações contribui para a validade interna dos achados, ainda que o tamanho reduzido da amostra impossibilite a generalização desses resultados para a totalidade dos usuários do serviço e/ou para outras populações. Além disso, cabe ressaltar que o baixo índice de recusa e a saturação dos dados observada no período de coleta contribuem para atenuar efeitos do viés de seleção e ampliar a representatividade da amostra em relação aos pacientes em tratamento paliativo na instituição onde se desenvolveu a pesquisa. mais intimamente relacionadas à QV do que as condições físicas. Somos, assim, convocados a atuar efetivamente na identificação de vulnerabilidades e implementação de estratégias efetivas para assegurar níveis satisfatórios de saúde mental em ambos. Afinal, os resultados apresentados no presente estudo, complementados por outros presentes na literatura (Delalibera et al., 2015; Yoon et al. 2014), sugerem que um adequado manejo dos sintomas ansiosos e depressivos, bem como estratégias para a diminuição da sobrecarga do cuidador, podem se relacionar a um aumento na qualidade de vida e saúde mental dos familiares cuidadores, melhorando a qualidade do cuidado prestado por esses e, ainda, a satisfação do paciente com o ambiente em que se encontra inserido.

Uma robusta revisão realizada por Northouse, Williams, Given e McCorkle (2012) a partir de cinco meta-análises, no intuito de investigar o impacto do câncer no bem-estar de familiares cuidadores e a efetividade de intervenções testadas para atenuar eventuais prejuízos, corroborou o desenvolvimento de distúrbios psicológicos como um importante impacto causado pelo câncer. A partir de sua busca, identificam três tipos de intervenção como predominantes e com efeitos positivos tanto para pacientes quanto para cuidadores, quais sejam: Psicoeducacional, Treinamento de habilidades e Aconselhamento terapêutico. Entretanto, conforme destacam os autores, apesar dos benefícios decorrentes de tais intervenções, elas raramente são incorporadas à assistência.

Os resultados aqui expostos devem ser vistos com cautela, em função das limitações do presente estudo. Dentre elas, destacamos (a) o tamanho reduzido da amostra, que se torna potencialmente problemático em função da sensibilidade dos testes de correlação; (b) a seletividade dos participantes, uma vez que apenas pacientes e cuidadores com maior crença na eficácia da Psicologia podem ter aceitado participar do estudo e, ainda, que estar presente na sessão de quimioterapia já possa indicar maior implicação no tratamento ou melhores condições de saúde física e mental; (c) problemas inerentes às medidas, como efeitos de desejabilidade social (tendência a responder de acordo com o que se considera socialmente aceito) e distorções de memória (dificuldade em lembrar-se com exatidão de eventos e/ou sentimentos pregressos).

Não obstante, convém pontuar que o grau de magnitude moderado a forte encontrado em várias correlações contribui para a validade interna dos achados, ainda que o tamanho reduzido da amostra impossibilite a generalização desses resultados para a totalidade dos usuários do serviço e/ou para outras populações. Além disso, cabe ressaltar que o baixo índice de recusa e a saturação dos dados observada no período de coleta contribuem para atenuar efeitos do viés de seleção e ampliar a representatividade da amostra em relação aos pacientes em tratamento paliativo na instituição onde se desenvolveu a pesquisa.

Apesar das limitações, acreditamos que o trabalho contribui para ampliar o conhecimento acerca da relação entre a qualidade de vida e o sofrimento psíquico em pacientes e seus familiares cuidadores, fornecendo dados que podem subsidiar discussões voltadas para o planejamento da assistência paliativa para esta população. Para estudos futuros, recomendamos a ampliação da amostra, bem como a realização da coleta em diferentes instituições, como forma de ampliar a possibilidade de generalização dos dados encontrados. Adicionalmente, sugerimos que a coleta de dados clínicos seja realizada também junto aos pacientes, a fim de confrontar tais dados com aqueles fornecidos pelos familiares e acessar o quanto esses pacientes são 
verdadeiramente informados acerca de sua doença e prognóstico, e que sejam explorados também os aspectos qualitativos da relação e vínculo entre o paciente e o cuidador, avaliando o efeito de tais aspectos na interação entre as manifestações psicopatológicas de ambos. Por fim, a realização de estudos longitudinais com esta população poderia indicar em que medida a progressão dos estágios do adoecimento atua para aumentar ou diminuir a congruência do par familiar-cuidador e, ainda, a forma como a sobrecarga do cuidador opera nestes diferentes estágios.

Em síntese, destacamos que, a despeito da ausência de correlação, a proximidade entre os escores das avaliações de pacientes e cuidadores indica que ambos apresentam níveis similares de Ansiedade, Depressão e QV, tornando-se importantes alvos do cuidado. Ansiedade, Depressão e Sobrecarga são sublinhadas como áreas nas quais pacientes e cuidadores podem se beneficiar de intervenções para melhorar sua QV. Reiteramos a importância da integração dos Cuidados Paliativos à assistência oncológica e também à formação dos profissionais de saúde, uma vez que sua filosofia prevê a consecução de atividades nos diferentes níveis de atenção à saúde e uma atuação efetivamente interdisciplinar, que se faz indispensável para abarcar as complexas demandas impostas por pacientes fora de possibilidades de cura e seus familiares cuidadores, sejam elas de ordem física, social, emocional e/ou espiritual.

\section{Referências}

Abrahão, S.S., Ricas, J., Andrade, D. F., Pompeu, F.C., Chamahum, L., Araújo, T.M., ... Lima, E.M. (2010). Dificuldades vivenciadas pela família e pela criança/adolescente com doença renal crônica. Jornal Brasileiro de Nefrologia, 32(1), 18-22. doi: https://dx.doi. org/10.1590/S0101-28002010000100004

Antonechen, A.C., \& Dóro, M.P. (2016). Qualidade de Vida, Ansiedade e Depressão em pacientes da hemato-onco com dor crônica. Saúde (Santa Maria), 42(1), 225-234. doi: http://dx.doi.org/10.5902/2236583419001

Borges, E.L., Franceschini, J., Costa, L.H.D., Fernandes, A.L.G., Jamnik, S., \& Santoro, I.L. (2017). Sobrecarga do cuidador familiar: a sobrecarga de cuidar de pacientes com câncer de pulmão, de acordo com o estágio do câncer e a qualidade de vida do paciente. Jornal Brasileiro de Pneumologia, 43(1), 18-23. doi: https://dx.doi. org/10.1590/s1806-37562016000000177

Botega, N.J., Bio, M.R., Zomignani, M.A., Garcia Junior, C., \& Pereira, W.A.B. (1995). Transtornos do humor em enfermaria de clínica médica e validação da escala de medida (HAD) de ansiedade e depressão. Revista de Saúde Pública, 29(5), 35563. doi: https://dx.doi.org/10.1590/S0034-89101995000500004

Bovero, A., Leombruni, P., Miniotti, M., Rocca, G., \& Torta R. (2016). Spirituality, quality of life, psychological adjustment in terminal cancer patients in hospice. European Journal of Cancer Care, 25, 961-969. doi: https://doi.org/10.1111/ecc.12360

Delalibera, M., Presa, J., Barbosa, A., \& Leal, I. (2015). Sobrecarga no cuidar e suas repercussões nos cuidadores de pacientes em fim de vida: revisão sistemática da literatura. Ciência e Saúde Coletiva, 20(9), 2731-2747. doi: https://dx.doi. org/10.1590/1413-81232015209.09562014

Ferrario, S.R., Cardillo, V., Vicario, F., Balzarini, E., \& Zotti, A.M. (2004). Advanced cancer at home: caregiving and bereavement. Palliative Medicine. 18(2), 129-136. doi: https://doi.org/10.1191/0269216304pm870oa

Fleck, M.P.A., Louzada, S., Xavier, M., Chachamovich, E., Vieira, G., Santos, L., \& Pinzon, V. (2000). Aplicação da versão em português do instrumento abreviado de avaliação da qualidade de vida "WHOQOL-bref". Revista de Saúde Pública, 34(2), 178183. doi: https://dx.doi.org/10.1590/S0034-89102000000200012 
Genezini, D., Castro, L., \& Rossi, S.G. (2009). Família, Adoecimento e Luto. In Veit, M. T. (org.) Transdisciplinaridade em Oncologia: caminhos para um atendimento integrado. São Paulo: HR Gráfica e Editora.

Goldstein, N.E., Concato, J., Fried, T.R., Kasl, S.V., \& Johnson-Hurzeler, R.B.E. (2004). Factors associated with caregiver burden among caregivers of terminally ill patients with cancer. Journal of Palliative Care, 20(1), 38-43. doi: https://doi. org/10.1177/082585970402000108

Götze, H., Brähler, E., Gansera, L., Polze, N., \& Köhler, N. (2014). Psychological distress and quality of life of palliative cancer patients and their caring relatives during home care. Support Care Cancer, 22, 2775-2782. doi: http://dx.doi-org.ez25.periodicos.capes.gov.br/10.1007/s00520-014-2257-5

Hodges, L.J., Humphris, G.M., \& Macfarlane, G. (2005). A meta-analytic investigation of the relationship between the psychological distress of cancer patients and their carers. Social Science \& Medicine, 60, 1-12. doi: https://doi.org/10.1016/j. socscimed.2004.04.018

Lee, Y. J., Kim, J. E., Choi, Y.S., Hwang, I. C., Hwang, S. W., Kim, Y. S., ... Kim, S. J. (2016). Quality of life discordance between terminal cancer patients and family: a multicenter study. Support Care Cancer, 24(7), 2853-2860. doi: http://dx.doi-org.ez25.periodicos.capes.gov.br/10.1007/s00520-016-3108-3

Li, Q., Xu, Y., Zhou, H., \& Loke, A. Y. (2016). Factors influencing the health-related quality of life of Chinese advanced cancer patients and their spousal caregivers: a cross-sectional study. BMC Palliative Care, 15, 72. http://doi.org/10.1186/ s12904-016-0142-3

Ministério da Saúde. (2014). Uma análise da situação de saúde e das doenças transmissíveis relacionadas à pobreza. Brasília - DF. Recuperado de: http://bvsms. saude.gov.br/bvs/publicacoes/saude brasil 2013 analise situacao saude.pdf

Neimeyer, R.A., \& Burke, L.A. (2012). Complicated grief and the end-of-life: Risk factors and treatment considerations. In R.A. Neimeyer \& L.A. Burke (editors), Counseling clients near the end-of-life (pp. 205-228). New York: Springer

Northouse, L., Williams, A.L., Given, B., \& McCorkle, R. (2012). Psychosocial Care for Family Caregivers of Patients with Cancer. Journal of Clinical Oncology, 30(11), 1227-1234. doi: https://ascopubs.org/doi/pdf/10.1200/JC0.2011.39.5798

Rezende, V.L., Derchain, S.F.M., Botega, N.J., Sarian, L.O., Vial, D.L., \& Morais, S.S. (2005). Depressão e ansiedade nos cuidadores de mulheres em fase terminal de câncer de mama e ginecológico. Revista Brasileira de Ginecologia e Obstetrícia, 27(12), 737-743. doi: https://dx.doi.org/10.1590/S0100-72032005001200006

Scazufca, M. (2002). Brazilian version of the burden interview scale for the assessment of burden of care in carers of people with mental illnesses. Revista Brasileira de Psiquiatria, 24, 12-17. doi: https://dx.doi.org/10.1590/S1516-44462002000100006

Song, J.I., Shin, D.W., Choi, J.Y., Kang, J., Baek, Y.J., Mo, H.N., ... Lee, O.K. (2012). Quality of life and mental health in the bereaved family members of patients with terminal cancer. Psychooncology, 21(11), 1158-1166. doi: https://doi-org. ez25.periodicos.capes.gov.br/10.1002/pon.2027

The Economist. (2015). The 2015 Quality of Death Index Ranking palliative care across the world: A report by The Economist Intelligence Unit. Recuperado de: http://www.lienfoundation.org/sites/default/files/2015\%20Quality\%20of\%20Death\%20Report.pdf

The WHOQOL Group. (1998). Development of the World Health Organization WHOQOL-bref. Quality of Life Assesment. Psychological Medicine, 28, 551-558. doi: https://doi-org.ez25.periodicos.capes.gov.br/10.1017/S0033291798006667

Zhang, B., Nilsson, M. E., \& Prigerson, H. G. (2012). Factors Important to Patients' Quality-of-Life at the End-of-Life. Archives of Internal Medicine, 172(15), 11331142. doi: http://doi.org/10.1001/archinternmed.2012.2364 
Wasner, M., Paal, P., \& Borasio, G.D. (2013). Psychosocial care for the caregivers of primary malignant brain tumor patients. Journal of Social Work at End Life \& Palliative Care, 9(1),74-95. https://doi.org/10.1080/15524256.2012.758605

World Health Organization (WHO). (2002). National cancer control programmers: polices and managerial guidelines (2a ed.). Geneva: WHO Press. Recuperado de: https://www.who.int/cancer/media/en/408.pdf

Worldwide Palliative Care Alliance (WPCA), \& World Health Organization (WHO). (2014). Global Atlas of Palliative Care at the End of Life. Recuperado de: https:// www.who.int/nmh/Global Atlas of Palliative Care.pdf

Yamashita, C.C. (2014). Avaliação de pacientes com câncer avançado e seus cuidadores: agrupamento de sintomas, qualidade de vida e sobrecarga. Tese de Doutorado, Fundação Antônio Prudente, São Paulo, SP, Brasil. Recuperado de: http://accamargo. phlnet.com.br/Doutorado/2014/CamilaCYamashita/CamilaCYamashita.pdf

Yoon, S.J., Kim, J.S., Jung, J.G., Kim, S.S., \& Kim, S. (2014). Modifiable factors associated with caregiver burden among family caregivers of terminally ill Korean cancer patients. Support Care Cancer, 22, 1243-1250. doi: http://dx.doi-org.ez25.periodicos.capes.gov.br/10.1007/s00520-013-2077-z 